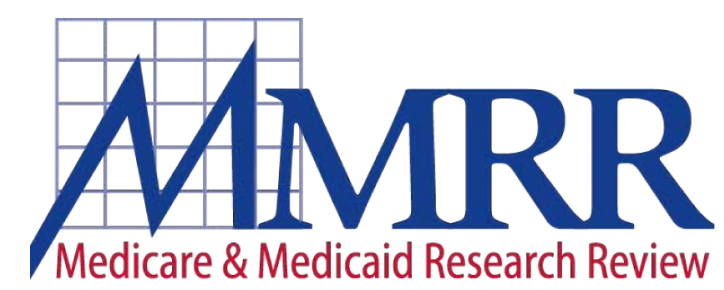

2014: Volume 4, Number 1

A publication of the Centers for Medicare \& Medicaid Services, Office of Information Products \& Data Analytics

\title{
Evaluating Whether Changes in Utilization of Hospital Outpatient Services Contributed to Lower Medicare Readmission Rate
}

\author{
Geoffrey Gerhardt, Alshadye Yemane, Keri Apostle, Allison Oelschlaeger, Eric Rollins, and \\ Niall Brennan
}

Centers for Medicare \& Medicaid Services

Objective: Descriptive analysis comparing changes in hospital inpatient readmissions to emergency department visits and observation stays that occurred within 30 days of an inpatient stay.

Population: Medicare fee-for-service (FFS) beneficiaries that had at least one acute hospital inpatient stay.

Data Source: Using 100 percent of claims in the Chronic Condition Data Warehouse, we compare growth in annual readmission stays to post-hospitalization emergency department visits and observation stays that were not accompanied by an inpatient stay. Comparisons are performed at the national level and within the Dartmouth Hospital Referral Regions (HRRs)

Results: In calendar year 2012, the national, all-cause, 30-day hospital readmission rate among Medicare FFS beneficiaries was 18.5 percent, a significant decline from 19 percent in 2011, which was also the average rate over the previous five years. The number of index admission stays per-1,000 Medicare beneficiaries declined by 4.3 percent, from 283.4 in 2011 to 271.3 in 2012.
On a per-1,000 beneficiary basis, the number of readmission stays declined by 6.8 percent, from 53.8 in 2011 to 50.1 in 2012. On the same per-beneficiary basis, the rate of outpatient visits to an emergency department occurring within 30 days of an index hospitalization remained similar at 23.5 in 2011 and 23.4 in 2012. Per-1,000 beneficiaries, the number of observation stays within 30 days of an index hospitalization increased by 0.3 percent, from 3.4 in 2011 to 3.7 in 2012.

Discussion: The reasons behind the decline in the Medicare readmission rate in 2012 are not yet clear. When looking at utilization changes in absolute terms, our findings suggest that the reduction in the nation-wide readmission rate observed in 2012 was not primarily the result of increases in either post-index ED visits or post-index observation stays.

Keywords: Medicare, hospitals, health policy, politics, law, regulation

ISSN: 2159-0354

doi: http://dx.doi.org/10.5600/mmrr.004.01.b03 


\section{Medicare \& Medicaid Research Review} 2014: Volume 4, Number 1

\section{Mission Statement}

Medicare \& Medicaid Research Review is a peerreviewed, online journal reporting data and research that informs current and future directions of the Medicare, Medicaid, and Children's Health Insurance programs. The journal seeks to examine and evaluate health care coverage, quality and access to care for beneficiaries, and payment for health services.

\section{http://www.cms.gov/MMRR/}

\section{U.S. Department of Health \& Human Services Kathleen Sebelius Secretary}

\section{Centers for Medicare \& Medicaid Services Marilyn Tavenner Administrator}

Editor-in-Chief

David M. Bott, Ph.D.

The complete list of Editorial Staff and Editorial Board members may be found on the MMRR Web site (click link): $\underline{\text { MMRR Editorial Staff Page }}$

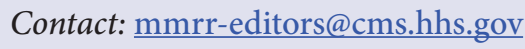

Published by the Centers for Medicare \& Medicaid Services.

All material in the Medicare \& Medicaid Research

Review is in the public domain and may be duplicated without permission. Citation to source is requested.

\section{Introduction}

Returning to the hospital shortly after an inpatient stay can be a traumatic experience for patients. Hospital readmissions can also be viewed as an indication of poor quality care and have been estimated to cost Medicare billions of dollars in potentially avoidable spending (Jencks, Williams, \& Coleman, 2009). Reducing readmissions has become a high priority for policy makers, providers, the Centers for Medicare \& Medicaid Services (CMS), and other payers. CMS is in the process of implementing several initiatives intended to better measure hospital readmissions, improve transitions of care once patients leave the hospital and post-hospitalization care, and ultimately reduce the number of readmissions that follow shortly after an inpatient stay (Kocher \& Adashi, 2011; Brock et al, 2013). These initiatives include public reporting mechanisms like Hospital Compare, community-based care improvement public-private partnerships like the Partnership for Patients, and payment reform like the Hospital Readmission Reduction Program (HRRP) and establishment of accountable care organizations.

We previously published data showing that the 30-day, all-condition readmission rate for Medicare fee-for-service (FFS) beneficiaries was significantly lower in 2012 compared to rates observed during the previous five years $(\mathrm{P}<.0001)$, dropping from an average of 19 percent over the 2007-2011 period to 18.5 percent in calendar year 2012 (Gerhardt et al., 2013). ${ }^{1}$ The cause of this decline is not yet clear, but some have speculated that reduced inpatient readmission rates in 2012 were the result of hospitals changing the way

\footnotetext{
${ }^{1}$ This reflects a revision to our previous report, which showed the 2012 readmission rate as 18.4 percent. The change is due to the inclusion of more complete claims data for 2012.
} 
they treat patients who return to the hospital after an admission (Carlson, 2013). This theory holds that the reduction in inpatient readmission rates observed in 2012 may be less the result of improvements in care quality and more the result of hospitals systematically substituting outpatient emergency department (ED) and/or observation stays for inpatient readmission stays. In other words, beneficiaries may be returning to hospitals just as often as they did before, but they are being classified differently.

Several studies have examined the relationship between inpatient readmission stays and other types of hospital care. Rising and colleagues found that nearly one-quarter of inpatient discharges were followed within 30 days by a visit to the ED, and argued that including these types of visits in a measure of re-hospitalization would enhance opportunities to improve post-hospitalization care (Rising, White, Fernandez, \& Boutwell, 2013). In looking for opportunities to improve post-discharge care, a study published by the Dartmouth Atlas Project found large variation in the percentage of patients that had an ED visit within 30 days of an inpatient hospitalization at both the regional and hospital levels (Goodman, Fisher, \& Chang, 2011).

Others have looked at the relationship between inpatient hospitalizations and both ED visits and outpatient observation stays and recommended the development of a composite measure of unplanned care that would include post-hospitalization ED visits, observation stays, and inpatient readmissions (Baier et al., 2013). Some studies have argued that focusing incentives on reducing inpatient readmission stays might obscure efforts by hospitals to substitute observation stays, ED services, and other types of hospital outpatient care for some inpatient care, and could result in lower quality care and poorer outcomes (Feng, Wright, \& Mor, 2012; Naylor et al., 2012).
There are many reasons why hospital utilization trends may have changed over the study period, including initiation of the Hospital Readmission Reduction Program, which took effect in October 2011, policy changes such as the switch to Medicare Severity Diagnosis Related Groups (MS-DRGs) that started in October 2007, and increased use of Recovery Audit Contractors. Our analysis focused on evaluating whether outpatient services may have substituted for inpatient readmission stays by comparing utilization trends, rather than identifying and evaluating the possible impact of all changes on utilization, which is beyond the scope of this paper.

\section{Data and Methods}

We present data using claims from the Chronic Conditions Data Warehouse, which has 100 percent of Medicare claims for beneficiaries who are enrolled in the FFS program (CCW, 2013). We used claims from 2007 through 2012. The study population includes all Medicare beneficiaries enrolled in Part A, including those under the age of 65, who were not enrolled in Medicare Advantage for any part of the year. All per-beneficiary results were calculated by dividing the number of stays or visits by the mean monthly enrollment for the study population over a given calendar year. We included claims from all acute care hospitals paid under the inpatient prospective payment system and critical access hospitals, but excluded other types of inpatient facilities such as rehabilitation and psychiatric hospitals.

Inpatient hospital stays that started within 30 days of discharge following an index admission were classified as readmission stays. ${ }^{2}$ For our analysis,

\footnotetext{
${ }^{2} \mathrm{An}$ index admission is an inpatient hospital stay, excluding stays where the patient died.
} 
the readmission rate equals the number of stays classified as inpatient readmission stays divided by the number of index admissions in a given calendar year. Readmission stays were also treated as index admissions if the beneficiary did not die during that stay. We did not count inpatient stays where the beneficiary died while in the hospital as an index admission, but such stays could be counted as a readmission stay. We treated same-day transfers as part of a single stay and started the 30 -day period at the end of the combined stay.

For this study, the readmission rate encompasses all clinical diagnoses and includes all beneficiaries regardless of whether their readmission stay was to the same hospital or a different hospital than the one where they were first discharged. Instances where a patient was discharged "against medical advice" were included as index admissions. The results also include readmission stays that might have been planned in advance or may be considered unavoidable.

Emergency department visits were determined by claims that contained emergency room revenue center codes 0450-0459 as well those with emergency room professional fee code 0981. In order to identify ED visits that may have been substituted for inpatient readmission stays, we classified outpatient ED visits that were provided within 30 days of an earlier index admission, that did not otherwise have a readmission stay within that 30-day period, as post-index ED visits.

Observation care is a type of outpatient service that is defined by Medicare as "a well-defined set of specific, clinically appropriate services, which include ongoing short-term treatment, assessment, and reassessment that are furnished while a decision is being made regarding whether patients will require further treatment as hospital inpatients or if they are able to be discharged from the hospital." For this study, observation stays include outpatient claims that have a revenue center code of G0378 (observation hours) or G0379 (direct admission to observation), at least eight hours of observation care, and did not have an inpatient admission. ${ }^{3}$ Our count of observation stays includes claims where the beneficiary received observation services and an outpatient procedure. Observation stays that occurred within 30 days of an earlier inpatient index admission that did not otherwise have a readmission stay within that 30 day period are classified as post-index observation stays.

If more than one ED visit or observation stay occurred within 30 days of an index admission, only the first occurrence was counted toward our count of post-discharge utilization. Neither ED visits nor observation stays were treated as index admission stays. Although we looked at observation stays and ED visits separately in this study, there is significant overlap between the two services-in 2012 roughly 85 percent of claims for observation stays also had an ED revenue code, up from 78 percent in 2007. In addition to looking at national level data, we stratified results by the 306 hospital referral regions (HRRs) defined by the Dartmouth Health Atlas.

\section{Results}

Exhibit 1 summarizes how utilization of the various hospital services described above changed from 2007 to 2012. For each type of service, the table shows the absolute number of services furnished, the number of services furnished per-1,000 Medicare beneficiaries, and the year-over-year change in services per-1,000 beneficiaries, expressed in both absolute and percent terms.

Since we are looking across several years of data, presenting results on a per-beneficiary basis controls

\footnotetext{
${ }^{3}$ Only observation stays that exceeded eight hours are included because Medicare does not pay for stays that last for fewer than eight hours. We also did not view shorter observation stays, where Medicare made no payment, as a viable alternative to inpatient readmission stays.
} 
Exhibit 1. Utilization of Hospital Services, 2007-2012 (Stays and visits in thousands)

\begin{tabular}{|c|c|c|c|c|c|c|}
\hline & 2007 & 2008 & 2009 & 2010 & 2011 & 2012 \\
\hline Index admission stays & 11,395 & 11,213 & 10,734 & 10,789 & 10,743 & 10,439 \\
\hline Per-1,000 beneficiaries & 307.5 & 307.4 & 294.0 & 291.1 & 283.4 & 271.3 \\
\hline Absolute Change & & -0.1 & -13.4 & -2.9 & -7.7 & -12.1 \\
\hline Percent Change & & * & -4.4 & -1.0 & -2.6 & -4.3 \\
\hline Inpatient readmission stays & 2,142 & 2,132 & 2,054 & 2,057 & 2,038 & 1,928 \\
\hline Per- 1,000 beneficiaries & 58.3 & 58.4 & 56.3 & 55.5 & 53.8 & 50.1 \\
\hline Absolute Change & & 0.1 & -2.2 & -0.8 & -1.7 & -3.7 \\
\hline Percent Change & & 0.2 & -3.7 & -1.3 & -3.1 & -6.8 \\
\hline All ED visits & 20,674 & 20,923 & 21,156 & 21,949 & 22,708 & 23,397 \\
\hline Per-1,000 beneficiaries & 562.7 & 573.5 & 579.4 & 592.3 & 599.1 & 608.1 \\
\hline Absolute Change & & 10.8 & 5.9 & 12.8 & 6.9 & 9.0 \\
\hline Percent Change & & 1.9 & 1.0 & 2.2 & 1.2 & 1.5 \\
\hline Post-index ED visits & 816 & 823 & 829 & 862 & 889 & 899 \\
\hline Per-1,000 beneficiaries & 22.2 & 22.6 & 22.7 & 23.3 & 23.5 & 23.4 \\
\hline Absolute Change & & 0.4 & 0.1 & 0.6 & 0.2 & -0.1 \\
\hline Percent Change & & 1.6 & 0.6 & 2.5 & 0.8 & -0.4 \\
\hline All observation stays & 979 & 1,077 & 1,221 & 1,323 & 1,426 & 1,608 \\
\hline Per-1,000 beneficiaries & 26.6 & 29.5 & 33.4 & 35.7 & 37.6 & 41.8 \\
\hline Absolute Change & & 2.9 & 3.9 & 2.3 & 1.9 & 4.2 \\
\hline Percent Change & & 10.8 & 13.3 & 6.8 & 5.4 & 11.0 \\
\hline Post-index observation stays & 87 & 96 & 109 & 118 & 128 & 142 \\
\hline Per-1,000 beneficiaries & 2.4 & 2.6 & 3.0 & 3.2 & 3.4 & 3.7 \\
\hline Absolute Change & & 0.3 & 0.3 & 0.2 & 0.2 & 0.3 \\
\hline Percent Change & & 11.7 & 12.8 & 6.9 & 6.1 & 9.2 \\
\hline
\end{tabular}

NOTE: ${ }^{*}=$ More than zero, but less than -0.1 .

Components may not add to totals due to rounding.

SOURCE: CMS analysis of Medicare claims data for 2007-2012.

for changes in Medicare fee-for-service enrollment. We believe that focusing on absolute changes in per-beneficiary utilization is the most appropriate way to interpret the data because it provides a sense of proportion when comparing temporal changes among different types of service. But, we also provide changes in percentage terms so readers can compare trends on a relative basis, as well.

Exhibits 2 and 3 graphically illustrate how the hospital services examined by the study have changed over the 2007-2012 time period. Exhibit 1 shows the annual absolute change in three categories of post-hospitalization services examined by this study. Exhibit 3 shows the cumulative changes in utilization among all types of hospital services included in our analysis, relative to their utilization levels in 2007. The findings are explained in more detail below.

\section{Inpatient Stays and Readmissions}

We found that inpatient hospital stays declined over the 2007 to 2012 period. In nominal terms, the number of index admission stays declined from 11.4 million in 2007 to 10.4 million in 2012 
Exhibit 2. Annual Change in Post-Discharge Hospital Services Per 1,000 Beneficiaries, 2008-2012

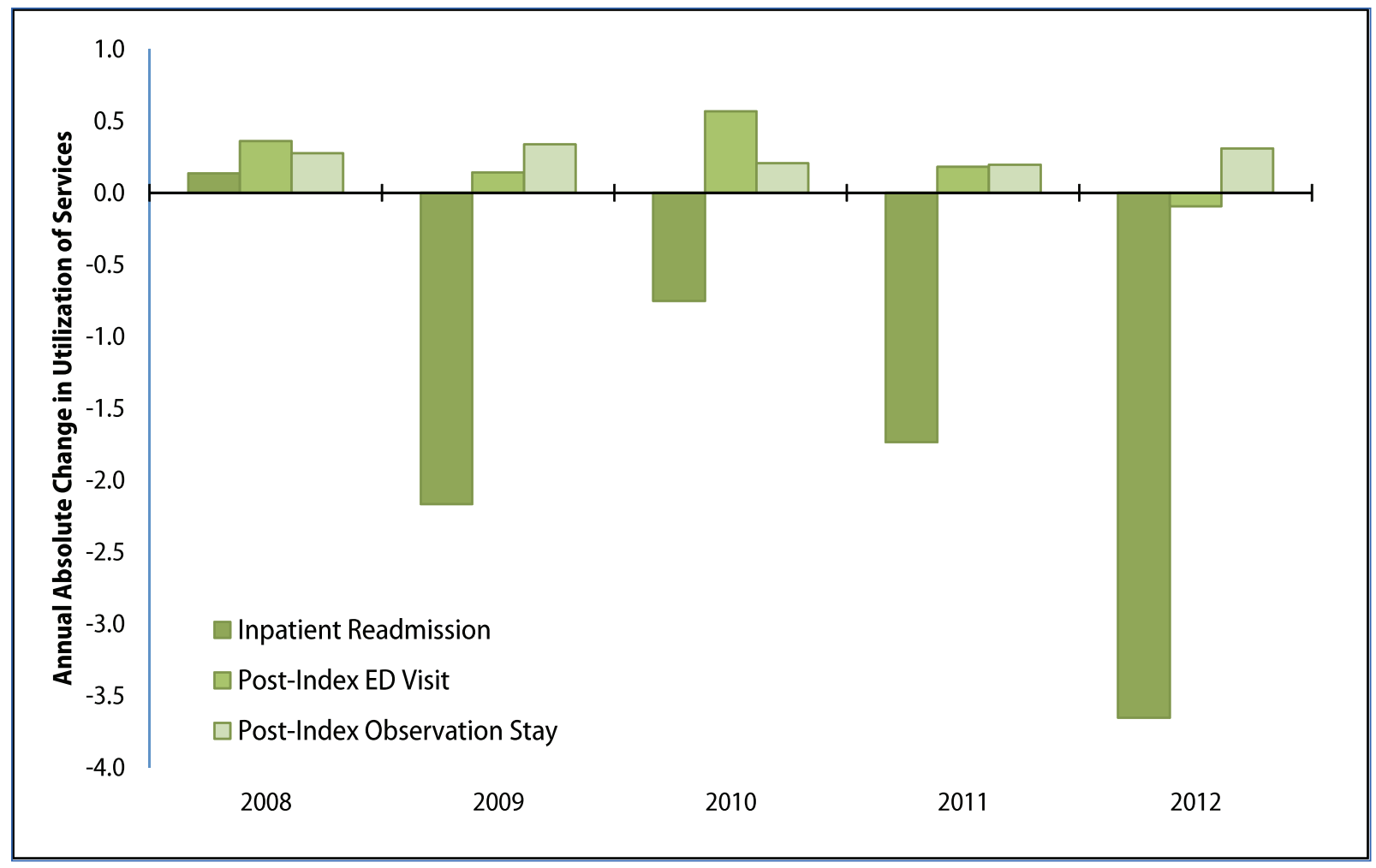

SOURCE: CMS analysis of Medicare claims data for 2007-2012.

(a 7.8 percent reduction), and readmission stays went from 2.1 million in 2007 to 1.9 million in 2012 (a 10 percent reduction). On a per-1,000 beneficiary basis, index admission stays declined from 307.5 in 2007 to 271.3 in 2012, while readmission stays dropped from 58.3 in 2007 to 50.1 in 2012. These observations are consistent with other studies that have shown declines in hospital inpatient admissions (MedPAC, 2013).

From 2007 to 2011, the reduction in index admissions was accompanied by proportionate declines in readmission stays. As such, the ratio of readmission stays to index admission stays remained relatively stable over that period, and the readmission rate held steady at around 19 percent. From 2011 to 2012, however, the number of readmission stays fell at a somewhat faster pace than the number of index admission stays. Per-1,000 beneficiaries, index admissions declined by 4.3 percent from 283.4 to 271.3 , while readmission stays went down by 6.8 percent, from 53.8 to 50.1. As a result, the 2012 readmission rate was 18.5 percent, which we found was a statistically significant reduction from the 2011 rate of 19.0 percent $(\mathrm{P}<.0001)$.

\section{Emergency Department Visits}

There were 23.4 million total hospital ED visits for Medicare FFS beneficiaries in 2012, and 16.0 million of them were classified as outpatient visits (i.e., they did not lead to an inpatient admission). Of the 16.0 million outpatient ED visits, nearly 900,000 , or just over 5 percent, were post-index ED visits.

On a per-beneficiary basis, the annual number of total ED visits climbed by 8 percent from 
Exhibit 3. Cumulative Change in Per-Beneficiary Utilization of Selected Hospital Services from 2007 Levels

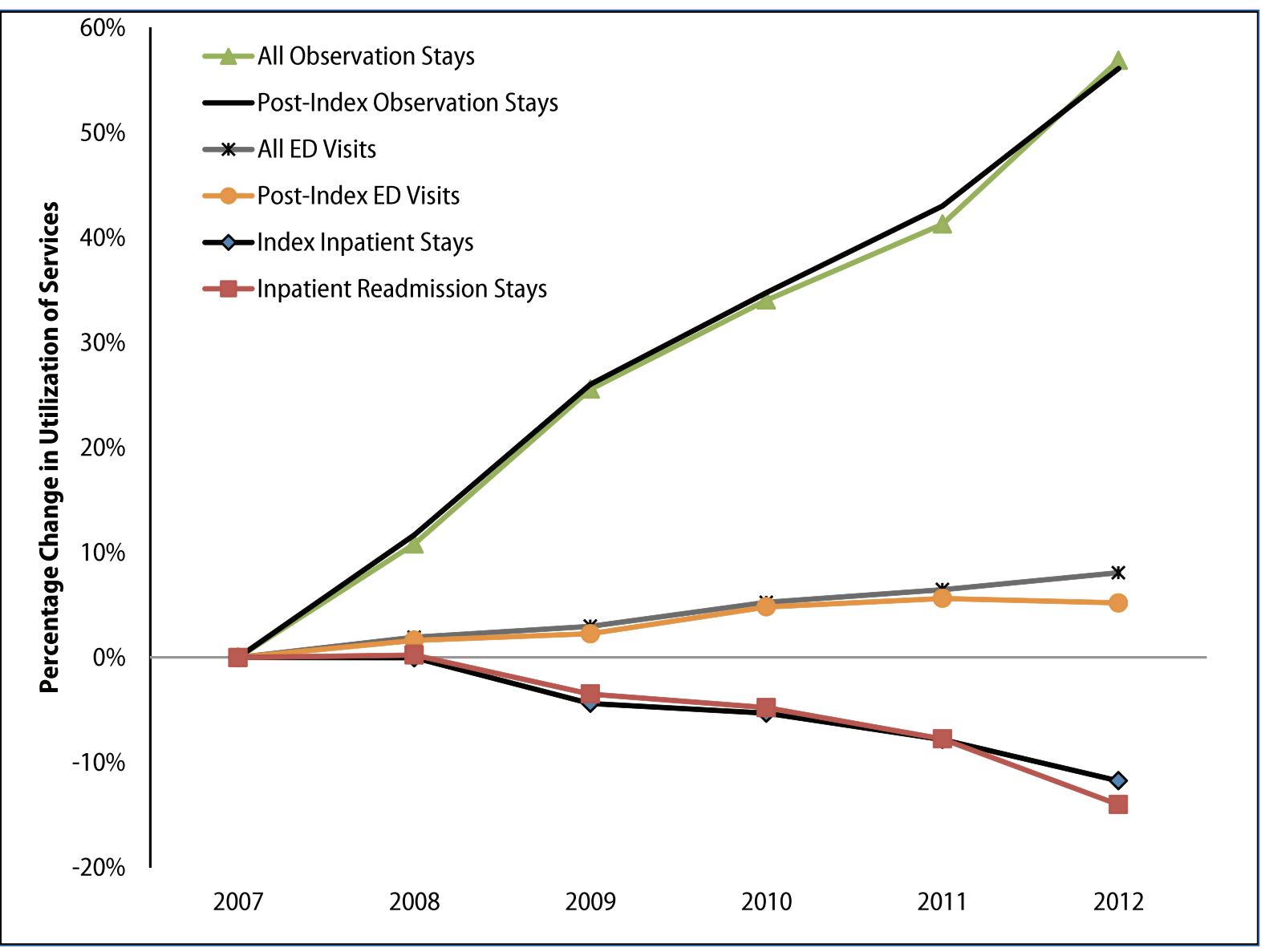

SOURCE: CMS analysis of Medicare claims data for 2007-2012.

2007 to 2012 (562.7 per-1,000 beneficiaries to 608.1), while ED visits that were outpatient only rose by 14 percent over the same period ( 364.0 per-1,000 beneficiaries to 416.0). Post-index ED visits displayed a similar growth pattern to total ED visits over most of the period covered by our analysis. From 2007 to 2011, the number of post-index ED visits per-1,000 beneficiaries rose from 22.2 to 23.5, an increase of 6 percent. However, between 2011 and 2012, post-index ED visits remained relatively flat at 23.4 per-1,000 beneficiaries, a decrease of just 0.4 percent. This comes in the presence of an 8 percent increase in total ED visits from 2007-2012 and follows several years of increases in post-index ED visits.
We also looked at how utilization of post-index ED visits changed at the HRR level from 2011 to 2012 (Exhibit 4). The one-year change in the number of post-index ED visits per-1,000 beneficiaries ranged from a high of 6.1 to -4.9 . There was a weak but positive relationship between the absolute change in post-index ED visits and the change in inpatient readmission stays, meaning that post-index ED visits had a tendency to fall ( -0.05 on average) in the same HRRs where readmission stays fell ( -3.3 on average). (These figures are the unweighted average of the HRR-level data and they differ somewhat from the national data shown in Exhibit 1.) Post-index ED visits increased and readmission stays decreased 
Exhibit 4. Change in Readmission Stays and Post-Discharge ED Visits Per 1,000 Beneficiaries, by HRR, 2011-2012

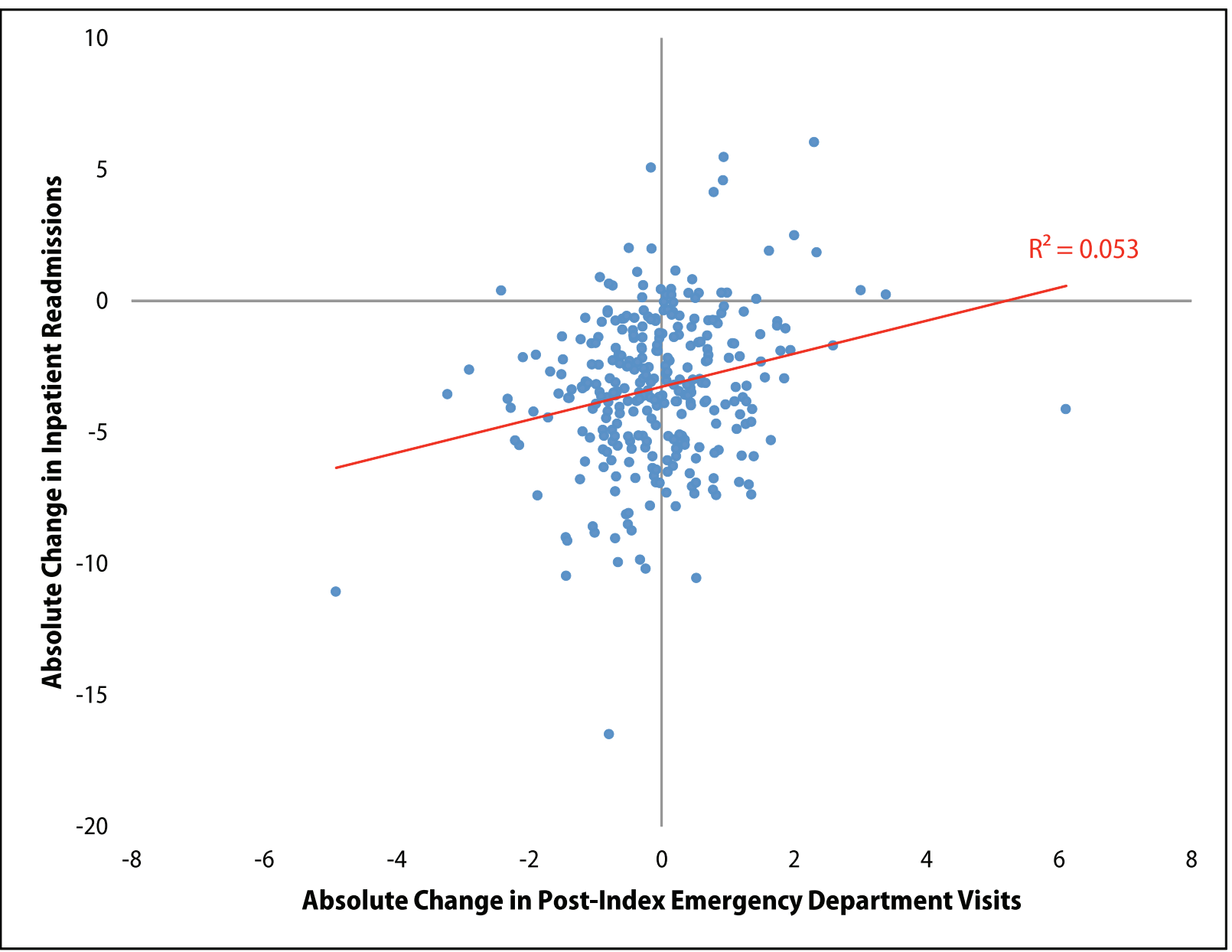

SOURCE: CMS analysis of Medicare claims data for 2007-2012.

in 118 out of the 306 HRRs. Among those 118 regions, the average per-1,000 beneficiary increase in post-index observation stays was 0.7 and the average change in readmission stays was -3.6 .

\section{Observation Stays}

Observation stays rose over the study period, going from 26.6 stays per- 1,000 beneficiaries in 2007 to 41.8 in 2012, a 57 percent increase. In 2007, we found there were 87,000 post-index observation stays, or 2.4 stays per-1,000 beneficiaries. In 2012, the total number of post-index observation stays had increased to 142,000 , or 3.7 stays per- 1,000 beneficiaries. The per beneficiary growth in post-index observation stays of 56 percent largely mirrored the overall increase in observation stays over the 2007 to 2012 period. Just looking at the change between 2011 and 2012, total observation staysgrewby 11 percent (37.6per-1,000 beneficiaries to 41.8 ), while post-index observation stays grew by 9.2 percent (3.4 per-1,000 beneficiaries to 3.7 ). The 2012 growth in post-index observation stays is largely in line with previous growth for those stays, which averaged just over 9 percent over the 2007-2011 period.

At the HRR level, the 2011 to 2012 change in post-index observation stays per-1,000 
Exhibit 5. Change in Readmission Stays and Post-Index Observation Stays Per-1,000 Beneficiaires, by HRR, 2011-2012

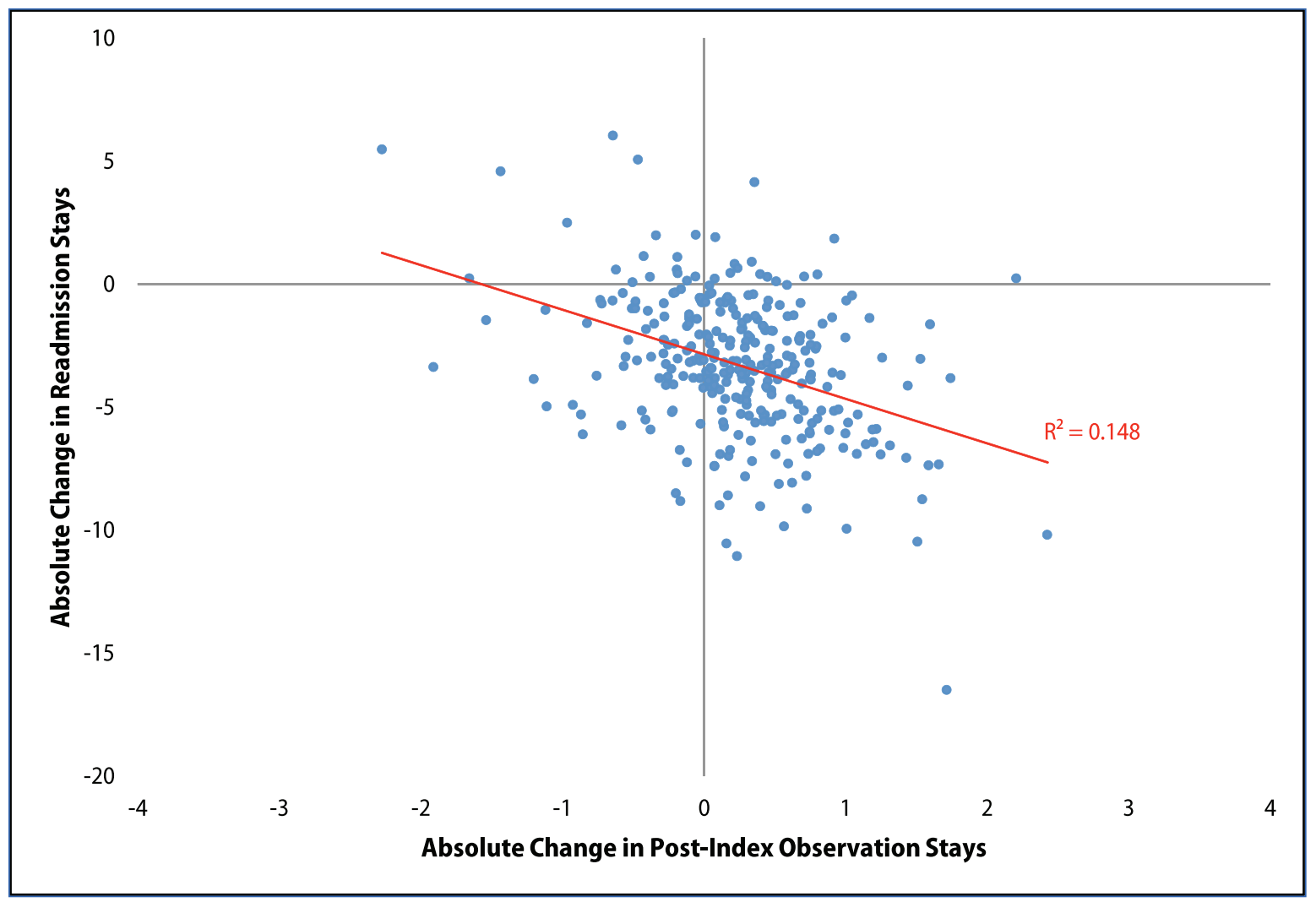

SOURCE: CMS analysis of Medicare claims data for 2007-2012.

beneficiaries ranged from a high of 2.4 to a low of -2.3 (Exhibit 5). The changes in readmission stays and post-index observation stays were inversely related, meaning that post-index observation stays had a tendency to rise in HRRs where readmission stays fell, but the average change in readmissions (-3.3) was much larger than the average increase in observation stays (0.2). (These figures are the unweighted average of the HRR-level data and they differ somewhat from the national data shown in Exhibit 1.) In the 200 HRRs that saw an increase in post-index observation stays and a decrease in readmission stays, the average increase in observation stays was 0.5 while the average decrease in readmission stays was -4.2 .

\section{Discussion}

The decline in hospital readmission rates observed in 2012 may indicate improvements in the quality of care delivered to Medicare beneficiaries. Preliminary unpublished data indicate that readmission rates continued to decline in 2013 . A comprehensive explanation for the decline in readmission rates is beyond the scope of this analysis and deserves further study.

When utilization is looked at on a per-beneficiary basis, our analysis of Medicare claims data does not suggest that the overall reduction in Medicare readmission rates that occurred in 2012 was primarily the result of greater use of outpatient ED visits or observation 
stays. We found the per-1,000 beneficiary prevalence of outpatient ED visits occurring within 30 days of an inpatient discharge largely held steady at 23.5 in 2011 and 23.4 in 2012. The prevalence of post-index observation stays increased from 3.4 per-1,000 beneficiaries in 2011 to 3.7 in 2012, a 9.2 percent increase that was in line with average annual growth of just over 9 percent for those types of stays over the previous five years. By comparison, the prevalence of inpatient readmission stays declined by 6.8 percent, from 53.8 per-1,000 beneficiaries in 2011 to 50.1 in 2012 .

In focusing on one possible explanation for the decline in readmission rates-that hospitals may have substituted post-index ED visits and/ or post-index observation stays for readmission stays-we have presented our data in terms of both absolute and percentage changes in per-capita utilization.

When looking at the question in absolute terms, our findings suggest that, at least at a national level, the reduction in readmission rates observed in 2012 was not primarily the result of increases in either post-index ED visits or post-index observation stays. If there had been a widespread substitution of readmission stays with post-hospitalization ED or observation stays in 2012, one would expect to see unusually large growth in utilization for those services in that year.

Post-index ED visits and post-index observation stays have increased over the past several years, but the absolute growth for these services did not increase in 2012 in ways that would suggest they systematically offset the decline in readmission stays. The size of the changes for post-index ED visits and observation stays in $2012(-0.1$ and 0.3 , respectively) were small relative to the decrease in readmission stays (-3.7), and largely consistent with utilization changes over the previous five years, whereas the decrease in readmission stays was substantially larger than decreases in previous years.

Comparisons of per-capita growth in percentage terms tells a similar story for post-index ED visits, which decreased by 0.4 percent in 2012, compared to a 6.8 percent decline in readmission stays. Post-index observation stays increased by 9.2 percent in 2012, which is larger than the percentage decline in readmission stays, but it is worth noting that the percentages represent 14,000 additional post-index observation stays and 110,000 fewer readmission stays.

Although our findings do not seem to indicate there was widespread, systemic substitution of outpatient services for inpatient readmission stays, this does not rule out the possibility that individual hospitals may have reduced inpatient readmission stays by increasing the use of post-index ED visits and/or observation stays. Our analysis at the HRR level suggests there could be some localized relationship between changes in utilization of inpatient readmission stays and outpatient services, but in general, any offsetting effect was relatively small. Exhibit 6 provides examples of the relationship between inpatient readmission stays and other post-index hospital services, using the 10 HRRs with the largest percentage point reductions in readmission rates from 2011 to 2012 and the 10 HRRs with the largest increases. The behavior of individual hospitals deserves more study with respect to the possible substitution of outpatient services for inpatient readmission stays, especially in cases where an inpatient stay may be more clinically appropriate. 
Exhibit 6. HRRs with Largest Changes in Readmission Rates in 2012

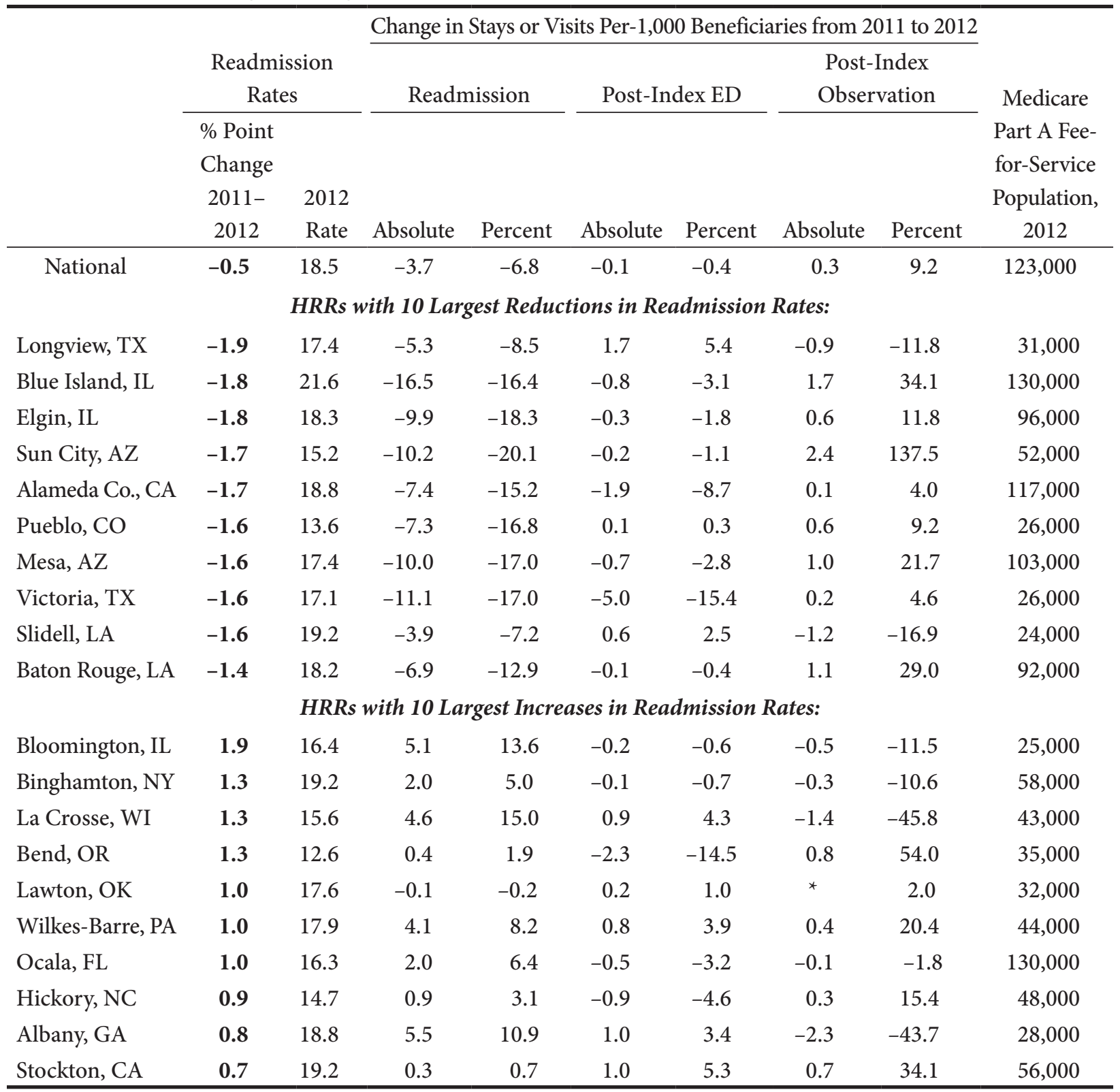

NOTE: ${ }^{*}=$ More than zero, but less than 0.1 .

SOURCE: CMS analysis of Medicare claims data for 2007-2012.

\section{Disclaimer}

The authors have been requested to report any funding sources and other affiliations that may represent a conflict of interest. The authors reported that there are no conflict of interest sources. The views expressed in this article are those of the authors and do not represent official policy of the Department of Health and Human Services.

\section{Correspondence}

Geoffrey Gerhardt, Centers for Medicare \& Medicaid Services, 200 Independence Ave., SW Washington, DC 20201, Geoff.Gerhardt@cms.hhs.gov T. (202) 260-0630

\section{Acknowledgment}

The authors would like to acknowledge the invaluable assistance of Brian O’Donnell and Daniel Gregory at General Dynamics Information Technology. 


\section{References}

Baier, R. R., Gardner, R., Coleman, E., Jencks, S., Mor, V., \& Gravenstein, S. (2013). Shifting the Dialogue from Hospital Readmissions to Unplanned Care. [Retrieved from http:// www.ajmc.com/publications/issue/2013/20131-vol19-n6/Shifting-the-Dialogue-FromHospital-Readmissions-to-Unplanned-Care/]. The American Journal of Managed Care, 19(6), 450-453. PubMed

Brock, J., Mitchell, J., Irby, K., Stevvens, B., Archibald, T., Goroski, A., \& Lynn, J. (2013, January). Association Between Quality Improvement for Care Transitions in Communities and Rehospitalizations Among Medicare Beneficiaries. Journal of the American Medical Association, 309(4), 381-391. Retrieved from http://jama.jamanetwork.com/article. aspx?articleid=1558278 PubMed http://dx.doi. org/10.1001/jama.2012.216607

CCW (Chronic Conditions Data Warehouse) (2013). [Research Database] Retrieved from http://www.ccwdata.org/

Carlson, J. (2013, June 8). Readmissions Are Down, but Observational-Status Patients Are Up-And That Could Skew Medicare Numbers. ModernHealthcare.com. Retrieved from http:// www.modernhealthcare.com/article/20130608/ MAGAZINE/306089991\# Feng, Z., Wright, B., \& Mor, V. (2012, June). Sharp Rise in Medicare Enrollees Being Held in Hospitals for Observation RaisesConcerns AboutCausesandConsequences. Health Affairs, 31(6). Retrieved from http:// content.healthaffairs.org/content/31/6/1251.full

Feng, Z., Wright, B., \& Mor, V. (2012, June). Sharp Rise in Medicare Enrollees Being Held in Hospitals for Observation Raises
Concerns About Causes and Consequences. [Retrieved from http://content.healthaffairs. org/content/31/6/1251.full]. Health Affairs, 31(6), 1251-1259. PubMed http://dx.doi. org/10.1377/hlthaff.2012.0129

Gerhardt, G., Yemane, A., Hickman, P., Oelschlaeger, A., Rollins, E., \& Brennan, N. (2013). Medicare Readmission Rates Showed Meaningful Decline in 2012. Medicare \& Medicaid Research Review, 3(2), E1-E12. Retrieved from http://www.cms.gov/ mmrr/Downloads/MMRR2013_003_02_b01.pdf http://dx.doi.org/10.5600/mmrr.003.02.b01

Goodman, D., Fisher, E., \& Chang, C. (2011). After Hospitalization: A Dartmouth Atlas Report on Post-Acute Care for Medicare Beneficiaries. Dartmouth Atlas Project \& Lake Research Group. Retrieved from http:// www.dartmouthatlas.org/downloads/reports/ Post_discharge_events_092811.pdf

Jencks, S. F., Williams, M., \& Coleman, E. (2009). Rehospitalizations Among Patients in the Medicare Fee-for-Service Program. The New England Journal of Medicine, 360(14), 1418-1428. Retrieved from http://www.nejm. org/doi/pdf/10.1056/NEJMsa0803563 PubMed http://dx.doi.org/10.1056/NEJMsa0803563

Kocher, R. P., \& Adashi, E. (2011, October). Hospital Readmission and the Affordable Care Act, Paying for Coordinated Quality Care. Journal of the American Medical Association, 306(16), 17941795. Retrieved from http://jama.jamanetwork. com/article.aspx?articleid=1104541 PubMed http://dx.doi.org/10.1001/jama.2011.1561

MedPAC (Medicare Payment Advisory Commission) (2013, June). A Data Book: Health Care Spending and the Medicare Program. Retrieved from http://medpac.gov/documents/ Jun13DataBookEntireReport.pdf 
Naylor, M. D., Kurtzman, E., Grabowski, D., Harrington, C., McClellan, M., \& Reinhard, S. (2012). Unintended Consequences of Steps to Cut Readmissions and Reform Payment May Threaten Care of Vulnerable Older Adults. Health Affairs, 31(7), 1623-1632. Retrieved from http://content.healthaffairs.org/ content/31/7/1623.full PubMed http://dx.doi. org/10.1377/hlthaff.2012.0110
Rising, K. L., White, L., Fernandez, W., \& Boutwell, A. (2013, April). Emergency Department Visits After Hospital Discharge: A Missing Part of the Equation. Annals of Emergency Medicine, 62(2), 145-150. Retrieved from http://www.annemergmed. com/article/S0196-0644(13)00096-6/ abstract PubMed http://dx.doi.org/10.1016/j. annemergmed.2013.01.024 